\title{
Optogenetic control of gut bacterial metabolism to promote longevity
}

2 Lucas A. Hartsough ${ }^{1}$, Matthew V. Kotlajich ${ }^{1}$, Bing Han ${ }^{3,7}$, Chih-Chun J. Lin ${ }^{3,4}$, Lauren Gambill²,

$5 \quad{ }^{1}$ Department of Bioengineering, ${ }^{2}$ Systems, Synthetic, and Physical Biology Program, Rice

6 University, Houston, TX; ${ }^{3}$ Huffington Center on Aging, ${ }^{4}$ Department of Molecular \& Human

7 Genetics, Baylor College of Medicine, Houston, TX; ${ }^{5}$ Howard Hughes Medical Institute;

$8 \quad{ }^{6}$ Department of Biosciences, Rice University; ${ }^{7}$ Current Address: Children's Hospital \& Institutes

9 of Biomedical Sciences, Fudan University, Shanghai, China, 201102 


\section{Abstract}

13 Gut microbial metabolism is associated with host longevity. However, because it requires

14 direct manipulation of microbial metabolism in situ, establishing a causal link between these two

15 processes remains challenging. We demonstrate an optogenetic method to control gene

16 expression and metabolite production from bacteria residing in the host gut. We genetically

17 engineer an Escherichia coli strain that synthesizes and secretes colanic acid (CA) under the

18 quantitative control of light. Using this optogenetically-controlled strain to induce CA production

19 directly in the Caenorhabditis elegans gut, we reveal the local effect of CA in protecting

20 intestinal mitochondria from stress-induced hyper-fragmentation. We also exploit different

21 intensities of light to determine that the lifespan-extending effect of CA is positively correlated

22 with its levels produced from bacteria. Our results show that optogenetic control offers a rapid,

23 reversible and quantitative way to fine-tune gut bacterial metabolism and uncover its local and

24 systemic effects on host health and aging. 


\section{Introduction}

Microbiome studies have identified correlations between bacteria and host aging (Kundu

31 heterogeneity of the human gut environment, these approaches are unable to elucidate how a

32 specific microbial species contributes to longevity. The nematode Caenorhabditis elegans has a

33 short and easily-measured lifespan, features that have revolutionized our understanding of the

34 molecular genetics of aging and longevity (Kenyon, 2010). Studies using C. elegans also provide

35 mechanistic insight into the association between bacterial species and host longevity (Gusarov et

36 al., 2013; Kim, 2013). Importantly, recent studies have revealed that bacterial metabolism can

37 produce specific products to directly influence the aging process in the host C. elegans or

38 modulate the effects of environmental cues on C. elegans lifespan (Cabreiro et al., 2013; Pryor et

39 al., 2019; Virk et al., 2016). These findings highlight the significance of bacterial metabolism in

40 regulating host physiology during the aging process and have inspired interest in directly

41 manipulating bacterial metabolism in situ in the host gastrointestinal (GI) tract.

In several recent studies, researchers have administered antibiotic- or carbohydrate-based

43 small molecule inducers to modulate gene expression from gut bacteria (Kotula et al., 2014; Lim

44 et al., 2017; Mimee et al., 2015). While this approach has enabled the in situ analysis of a gut

45 bacterial-host interaction (Lim et al., 2017), chemical effectors may have unwanted side-effects

46 on host or microbial physiology and subject to slow and poorly-controlled transport and

47 degradation processes that ultimately limit their precision. 
Optogenetics combines light and genetically-engineered photoreceptors to achieve unrivaled control of biological processes (Olson and Tabor, 2014). Previously, we and others have engineered bacterial photoreceptors that activate or repress gene expression in response to

51 specific wavelengths of light (Levskaya et al., 2005; Li et al., 2020; Ohlendorf et al., 2012; Ong

52 and Tabor, 2018; Ong et al., 2017; Ramakrishnan and Tabor, 2016; Ryu and Gomelsky, 2014;

53 Schmidl et al., 2014). These photoreceptors have been used to achieve precise quantitative

54 (Olson et al., 2017, 2014), temporal (Chait et al., 2017; Milias-Argeitis et al., 2016; Olson et al.,

55 2017, 2014), and spatial (Chait et al., 2017; Levskaya et al., 2005; Ohlendorf et al., 2012; Tabor

56 et al., 2010) control of bacterial gene expression in culture conditions. They have also been used

57 to characterize and control transcriptional regulatory circuits (Chait et al., 2017; Olson et al.,

58 2014; Tabor et al., 2009) and bacterial metabolic pathways (Fernandez-Rodriguez et al., 2017;

59 Tandar et al., 2019) in vitro. Here, we hypothesized that optogenetic control of bacterial gene

60 expression might provide a new way to manipulate bacterial metabolism in vivo in the host GI

61 tract, with high temporal and spatial precision and no unwanted side-effects.

We address this possibility using the E. coli-C. elegans interaction model, a testable

63 system with known mechanistic links between bacterial metabolism and host longevity and with

64 complete optical transparency. In particular, CA is an exopolysaccharide synthesized and

65 secreted from $E$. coli, which can extend the lifespan of the host $C$. elegans through modulating

66 mitochondrial dynamics (Han et al., 2017). We thus have genetically engineered an E. coli strain

67 to put its biosynthesis of CA under a switchable control between green and red lights, and then

68 utilized green light to induce CA production from this strain in the gut of the host C. elegans. We

69 discovered that light-induced CA from bacteria residing in the host is sufficient to modulate

70 mitochondrial dynamics and lifespan, which gives even more potent effects than dietary 
71 supplementation of CA. Furthermore, this optogenetic manipulation allowed us to investigate the

72 local effect of CA on intestinal cells in a time-controlled manner and its systemic effect on

73 organisms in a quantitative way, which are not possible with purified CA or CA-overproducing

74 genetic mutants. This work paves the road for future application of bacterial optogenetics in

75 understanding bacteria-host interaction with temporal, spatial and quantitative controls and

76 minimal chemical interference.

\section{Results}

To demonstrate optogenetic control over gut bacterial gene expression, we first engineered E. coli strain LH01, wherein our previous light-switchable two-component system

81 CcaSR (Schmidl et al., 2014) controls expression of superfolder green fluorescent protein ( $s f g f p)$,

82 and mcherry is expressed constitutively to facilitate identification of the bacteria (Fig. 1a,

83 Supplementary Fig. 1, Supplementary Tables 1-3). In LH01, green light exposure switches

$84 \mathrm{CcaS}$ to an active state in the presence of chromophore phycocyanobilin (PCB), wherein it

85 phosphorylates the response regulator CcaR. Phosphorylated CcaR then activates transcription of

$86 s f g f p$ from the $\mathrm{P}_{c p c G 2-172}$ output promoter. Red light reverts active CcaS to the inactive form, de-

87 activating $s f g f p$ expression (Fig. 1a).

88 We then reared two groups of $C$. elegans from the larval to the adult stage on plates of

89 LH01 under red or green light, respectively (Fig. 1b). Next, we washed away external bacteria,

90 applied the paralyzing agent levamisole to prevent expulsion of gut contents, and transferred the

91 worms to agar pads. Finally, we switched the light color from red to green, or green to red, and

92 used epi-fluorescence microscopy to image the resulting changes in fluorescence in the gut

93 lumen over time (Fig. 1c). In the red-to-green (step ON) experiment, we observed that sfGFP 
94 fluorescence in the worm gut lumen starts low, begins to increase within 2 hours, and reaches a

95 saturated high level at 6 hours (Fig. 1d). In contrast, in the green-to-red (step OFF) experiment,

96 sfGFP fluorescence begins high, and decreases exponentially between hours 1-7 (Fig. 1d). This

97 light response is abolished when the PCB biosynthetic operon is removed $(\triangle \mathrm{PCB})($ Fig. 1d),

98 demonstrating that sfGFP levels are specifically controlled by CcaSR.

99 Next, we used flow cytometry to achieve high-throughput single-cell resolution analysis

100 of this in situ bacterial light response. Specifically, we reared worms in red and green light as

101 before, but then washed, paralyzed, and placed them into microtubes prior to light switching

102 (Fig. 1b). At several time points over the course of 8 hours, we homogenized the animals,

103 harvested the gut contents, and sorted bacterial cells and measured fluorescence via cytometry.

104 This experiment revealed that bacteria in the host gut remain intact (Supplementary Fig. 2) and

105 respond to light in a unimodal fashion (Fig. 1e-g). Furthermore, the temporal dynamics of the

106 gene expression response and dependence on $\mathrm{PCB}$ recapitulate our microscopy results

107 (Supplementary Fig. 3). We also confirmed that residual bacteria on the exterior of worms do

108 not contribute to the flow cytometry measurements (Supplementary Fig. 4). Together, these

109 experiments demonstrate that we can use optogenetics to rapidly, reversibly induce gene

110 expression of E. coli residing in the C. elegans gut.

111 Next, we sought to utilize our optogenetic method to modulate the production of specific

112 metabolites in bacteria residing in the gut of live hosts. It is well-known that bacterial genes

113 involved in the same metabolic process are often clustered into operons and co-regulated at the

114 transcriptional level. We took advantage of this coordinated mode of regulation and chose the

115 cps operon and its transcription activator RcsA for testing optogenetic control of bacterial

116 metabolism. The cps operon in E. coli consists of 19 genes that encode enzymes required for the 
117 biosynthesis and secretion of CA (Torres-Cabassa and Gottesman, 1987), and CA-overproducing

118 bacterial mutants $\Delta l o n$ and $\Delta h n s$ promote longevity in the host C. elegans (Han et al., 2017). To

119 place CA biosynthesis under optogenetic control, we engineered an E. coli strain (MVK29)

120 lacking genomic $\operatorname{rcs} A$ and expressing a heterologous copy of $r$ csA under the control of CcaSR

121 (Fig. 2a, Supplementary Tables 1-3). We first examined whether MVK29 could respond to

122 green light and induce CA production and secretion. To this end, we grew the strain in batch

123 culture under red or green light and quantified supernatant CA levels. In red light, MVK29

124 secretes CA to concentrations below the limit of detection of the assay, similar to the $\Delta \operatorname{rcs} A$

125 mutant (Fig. 2b). Green light, on the other hand, induces MVK29 to secrete high levels of CA,

126 and removal of the PCB biosynthetic operon abolishes this response (Fig. 2b). Moreover,

127 mutation of the $\mathrm{CcaS}$ catalytic histidine to a non-functional alanine (H534A), or the CcaR

128 phosphorylation site from an aspartic acid to a non-functional asparagine (D51N) abolishes

129 detectable CA production (Fig. 2b). Importantly, the level of secreted CA increases sigmoidally

130 with green light intensity, similar to the response of CcaSR itself (Fig. 2c) (Schmidl et al., 2014).

131 We conclude that we have placed CA production under the control of the CcaSR system, and 132 that we can use light to tune the production of bacterial metabolites.

133 We then used this approach to study a gut bacterial metabolite-host interaction pathway 134 in vivo. We first reared worms expressing mitochondrially-localized GFP (mito-GFP)

135 (Supplementary Table 3) on MVK29 with red light. We then continued this red-light exposure 136 for one group, and switched a second to green, for an additional 6 hours, and immediately 137 imaged intestinal cell mitochondrial morphology using confocal microscopy (Fig. 3b). We found 138 that mitochondrial fragmentation increases in worms exposed to the bacteria with light-induced 
139 CA secretion (Fig. 3c). This result recapitulates the phenotype observed in worms supplemented 140 with purified CA (Han et al., 2017).

141 Next, we induced CA production directly from bacteria residing within the host gut. To

142 this end, we paralyzed the worms carrying mito-GFP, split them into two groups, and treated one

143 with red and the second with green for 6 hours (Fig. 3a). We then analyzed mitochondrial

144 morphology in intestinal cells using confocal microscopy. We found that the 6-hour levamisole

145 treatment does not kill worms but leads to mitochondrial hyper-fragmentation (Fig. 3d), which

146 might be due to the inhibitory effect of levamisole on mitochondrial NADH-oxidizing enzymes

147 (Köhler and Bachmann, 1978). This stress-induced effect resembles mitochondrial decay related

148 to aging and age-related neurodegenerative diseases (Cho et al., 2009; Exner et al., 2007;

149 Sebastián et al., 2017). Interestingly, we found that green light exposure counteracts this hyper-

150 fragmentation in paralyzed worms bearing MVK29 in the gut (Fig. 3d). Importantly, we found

151 no such effects in worms bearing the $\triangle \mathrm{PCB}, \mathrm{CcaS}(\mathrm{H} 534 \mathrm{~A}), \mathrm{CcaR}(\mathrm{D} 51 \mathrm{~N})$ or $\triangle \operatorname{rcs} A$ mutant

152 strains (Fig. 3d), suggesting that this protective effective is a result of light-induced CA

153 overproduction in the gut. These results not only show that optogenetics can be utilized to induce

154 CA secretion from gut-borne E. coli in vivo, but also reveal a local protective effect of CA on 155 intestinal cells.

156 Finally, we took the advantage of the quantitative control afforded by optogenetics to 157 investigate how the lifespan-extending effect of CA relates to CA levels. Beginning at the day-1 158 adult stage, we exposed worms bearing MVK29 to red, or two green light intensities resulting in

159 intermediate or high CA secretion, and measured their lifespans. We found that the lifespan 160 extension increases proportionally with green-light intensity (Fig. 4a), revealing the pro161 longevity effect of CA is dose dependent. We also noticed that the extent of lifespan extension 
162 by light is much stronger than that caused by dietary supplementation of purified CA (Han et al.,

163 2017), suggesting the high efficacy of optogenetic induction to modulate host physiology. As a

164 control, we repeated the experiment with worms bearing the CA-overproducing $\Delta$ lon mutant and

165 showed that the lifespan extension caused by $\Delta l o n$ is independent of light exposure (Fig. 4b).

166 The extent of lifespan extension is similar to the MVK29 intermediate green light condition, but

167 less than the MVK29 high green light condition (Fig. 4a, b), suggesting that MVK29 is capable

168 of producing higher levels of CA than $\Delta l o n$. In addition, the lifespan of worms bearing the $\Delta \operatorname{rcs} A$

169 mutant is also light-independent and is comparable to that of MVK29 worms under red light

170 (Fig. 4a, c). These results suggest that optogenetic control is sufficient to induce bacterial

171 production of pro-longevity compounds and improve host health, and can exert stronger

172 beneficial effects than administration of a bacterial mutant or supplementing purified

173 compounds. Importantly, unlike the traditional approach of introducing a bacterial mutant,

174 optogenetic control of bacterial metabolism can modulate a host-level phenotype in a 175 quantitative manner.

\section{Discussion}

Our method has broad applications for studying microbe-host interactions in situ. For example, we have identified about two dozen additional $E$. coli genes that are unrelated to CA

180 biosynthesis and that enhance worm longevity when knocked out (Han et al., 2017), though the

181 mechanisms by which they act remain largely unclear. By using light to induce their expression

182 in the gut and measuring acute host responses such as changes in mitochondrial dynamics, the

183 role of these genes in gut microbe-host interactions could be further explored. In another

184 example, the quorum-sensing peptide CSF and nitric oxide, both of which are produced by 
185 Bacillus subtilis during biofilm formation, have been found to extend worm lifespan through

186 downregulation of the insulin-like signaling pathway (Donato et al., 2017). We have recently

187 ported CcaSR into B. subtilis and demonstrated that it enables rapid and precise control of gene

188 expression dynamics (Castillo-Hair et al., 2019). The method we report here should enable in

189 situ studies of how gene expression and metabolite production from this important Gram-positive

190 model bacterium impact longevity as well. interaction pathways. Specifically, we and others have co-expressed CcaSR with independentlycontrollable blue/dark and red/far-red reversible light sensors in order to achieve simultaneous

194 and independent control of the expression of up to three genes in the same bacterial cell

195 (Fernandez-Rodriguez et al., 2017; Olson et al., 2017; Tabor et al., 2010). Such optogenetic

196 multiplexing could be performed in situ and used to study potential synergistic, antagonistic, or

197 other higher-order effects of multiple bacterial genes or pathways. A large number of eukaryotic

198 photoreceptors have also been developed, enabling optical control of many cell- and

199 neurobiological processes (Deisseroth, 2015; Gautier et al., 2014; Goglia and Toettcher, 2018;

200 Leopold et al., 2018). Bacterial and eukaryotic photoreceptors could be combined to enable

201 simultaneous optical manipulation of bacterial and host pathways in order to interrogate whether

202 or how they interact. Optogenetics could also be used to manipulate bacterial and/or host

203 pathways at specific locations within the gut to examine location- or tissue-dependent 204 phenomena.

Finally, our method could be extended to other bacteria or hosts. In particular, it should

206 be possible to port CcaSR or other bacterial photoreceptors into native C. elegans symbionts

207 (Zhang et al., 2017) or pathogens(Couillault and Ewbank, 2002). Because these strains stably 
208 colonize the host, the use of these bacteria could eliminate the need for paralysis, and facilitate

209 longer-term experiments. It is likely that light can also be used to control gut bacterial gene

210 expression in other model hosts such as flies, zebrafish, or mammals. Red-shifted wavelengths

211 and corresponding optogenetic tools(Ong et al., 2017; Ryu and Gomelsky, 2014) may prove

212 superior for less optically transparent or larger animals. Overall, by enabling precision control of

213 bacterial gene expression and metabolism in situ, we believe that optogenetics will greatly

214 improve our understanding of a wide range of microbe-host interactions.

\section{Acknowledgements}

217 The LED array used to illuminate C. elegans plates was designed by Brian Landry \&

218 Sebastián Castillo-Hair. The mounting hardware for the microscope LED array was designed by

219 Ravi Sheth. We thank Ravi Sheth for discussions during early stages of the project. We thank Dr.

220 Joel Moake for the use of his cytometer. This work was supported by the John S. Dunn

221 Foundation (J.J.T. and M.C.W.) and US National Institutes of Health, 1R21NS099870-01

222 (J.J.T.), DP1DK113644 (M.C.W.), R01AT009050 (M.C.W.). LAH was supported by a NASA

223 Office of the Chief Technologist Space Technology Research Fellowship (NSTRF

224 NNX11AN39H).

225

226 Author Contributions

227 JJT and MW conceived of the study. LAH and MVK designed experiments. MVK and LAH

228 constructed plasmids and strains. LAH, MVK, BH, CJL, LG, and MW performed experiments.

229 CJL and LG scored single-blinded mitochondrial confocal micrographs. LAH, MVK, and MW

230 analyzed and interpreted results. LAH, MW, and JJT wrote the manuscript. 
bioRxiv preprint doi: https://doi.org/10.1101/2020.02.25.964866; this version posted March 26, 2020. The copyright holder for this preprint (which was not certified by peer review) is the author/funder. All rights reserved. No reuse allowed without permission.

\section{Declaration of Interests}

233 The authors declare no competing interests.

234 


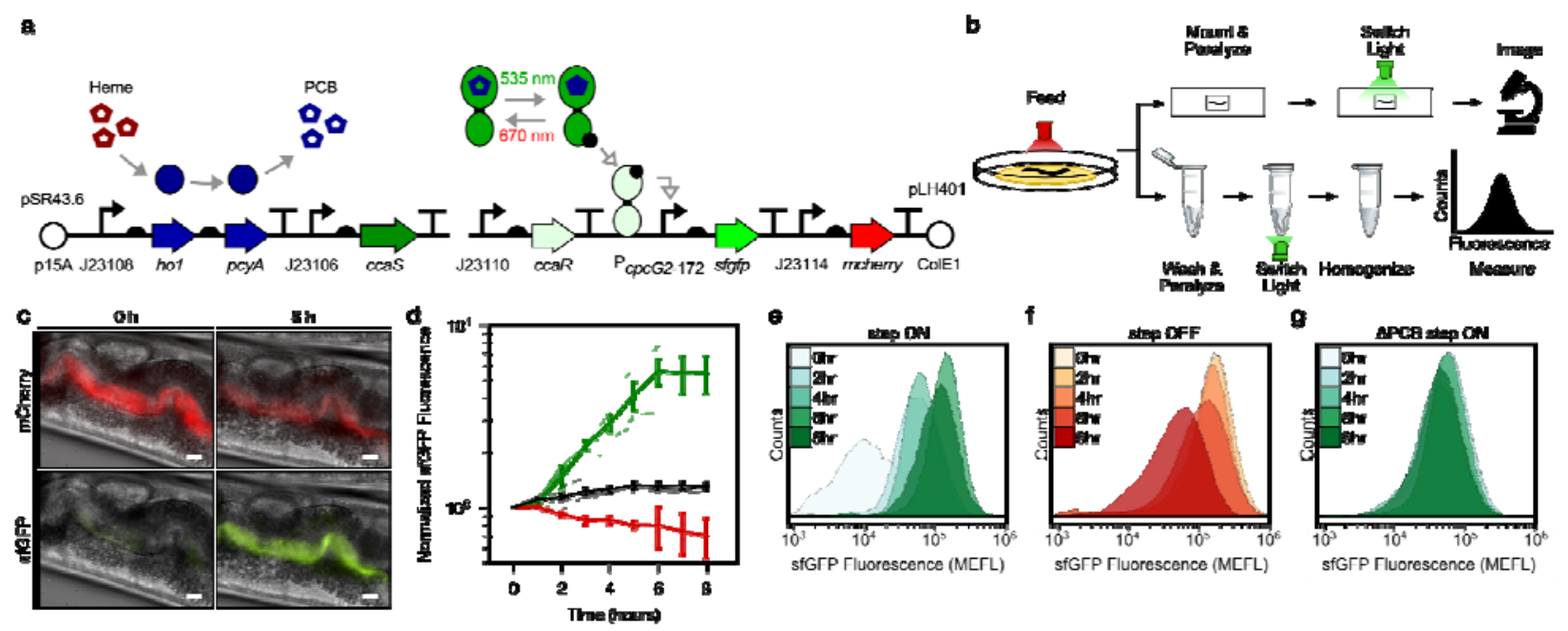

Figures and legends

237 Figure 1. Optogenetic control of $C$. elegans gut bacterial gene expression. (a) Strain LH01.

238 (b) Microscopy and cytometry workflows. (c) Fluorescence microscopy images 0 and $8 \mathrm{~h}$ after

239 green light exposure in the step ON experiment. Scale bar: $10 \mu \mathrm{m}$. (d) Response dynamics in the

240 step ON (green) and step OFF (red) microscopy experiments. Black: $\triangle \mathrm{PCB}$ strain (step ON

241 experiment). Individual- (light lines) and multi-worm average (dark lines) data are shown. $n=7$,

242 4, 6 worms for green, red, black data sets (measured over 2, 3, 1 days, respectively). Error bars:

243 SEM. (e-g) Flow cytometry histograms for response dynamics experiments. MEFL: molecules of 244 equivalent fluorescein. 

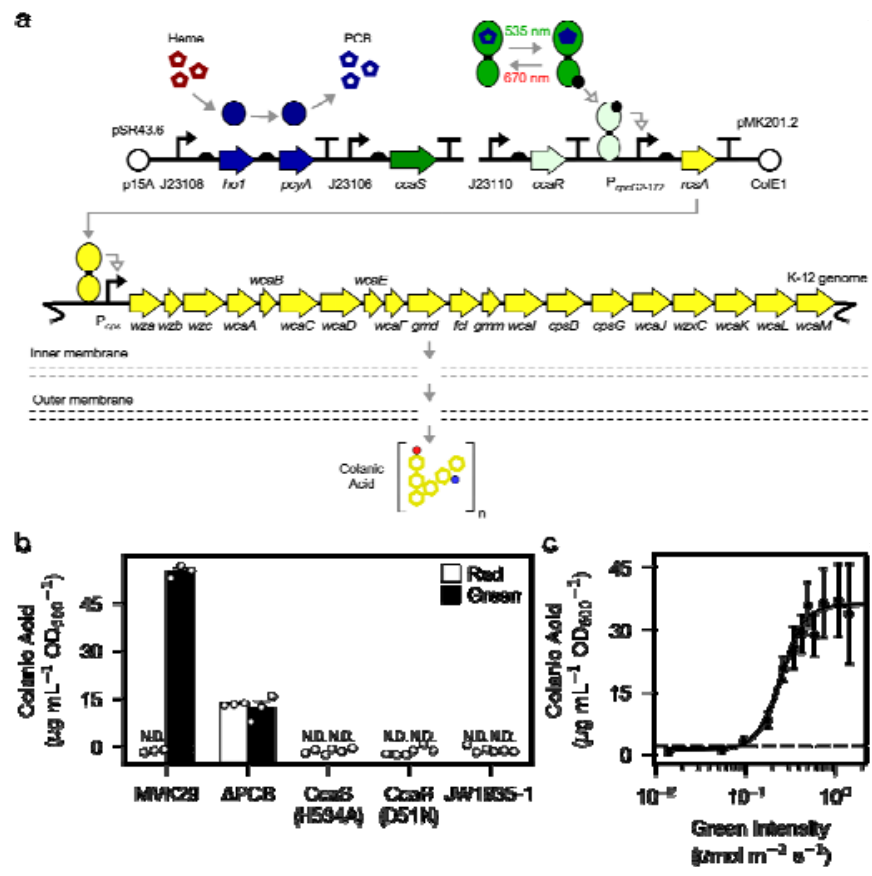

247 Figure 2. Optogenetic control of colanic acid biosynthesis. (a) Strain MVK29. (b) CA

248 secretion levels for MVK29 and control strains exposed to red and green light. JW1935-1 is the

249 E. coli rcsA background strain used in this study. N.D.: below assay limit of detection. (c)

250 Green light intensity versus CA secretion level for MVK29. Data points represent 3 biological

251 replicates collected on a single day. Dashed line: limit of detection. Error bars indicate standard

252 deviation of the three biological replicates. 


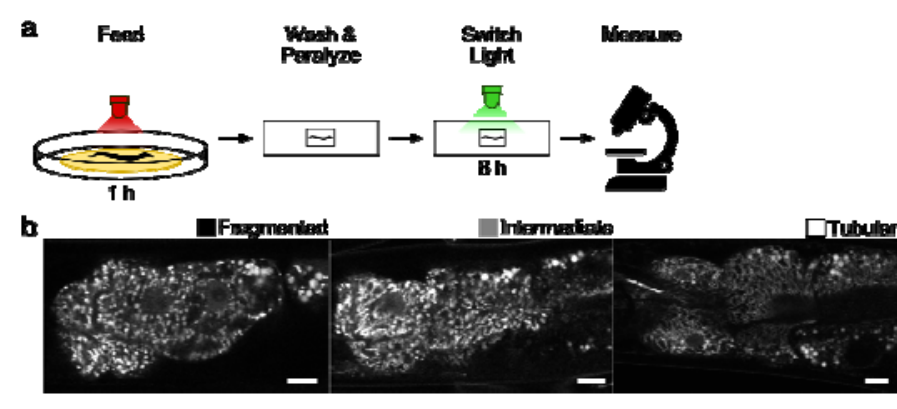

c

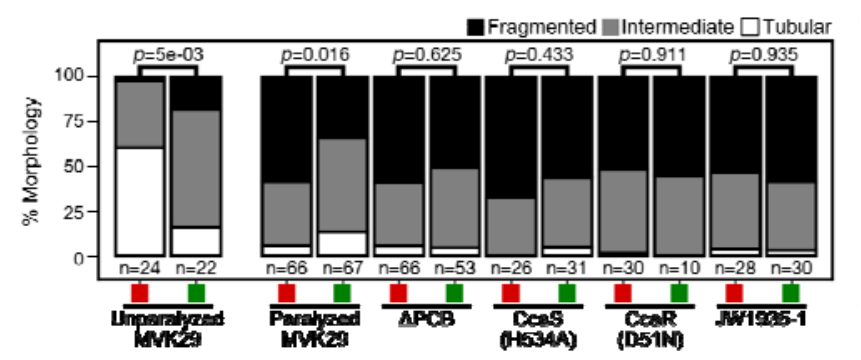

256 Figure 3. Light-regulated CA secretion modulates C. elegans mitochondrial dynamics. (a)

257 Schematic of experiment for activating CA biosynthesis in situ. (b) Representative images of the

258 mitochondrial network of anterior intestinal cells immediately distal to the pharynx are scored as

259 fragmented, intermediate, or tubular, as previously(Han et al., 2017). Scale bars: $10 \mu \mathrm{m}$. (c)

260 Mitochondrial fragmentation profiles of un-paralyzed worms fed MVK29 while exposed to red

261 or green light for $6 \mathrm{~h}$. (d) Fragmentation profiles for worms fed the indicated strain, then

262 paralyzed for $6 \mathrm{~h}$ while exposed to red or green light. The number of worms included in each

263 condition is indicated below each bar. The Chi-Squared Test of Homogeneity was used to

264 calculate $p$-values between conditions. 

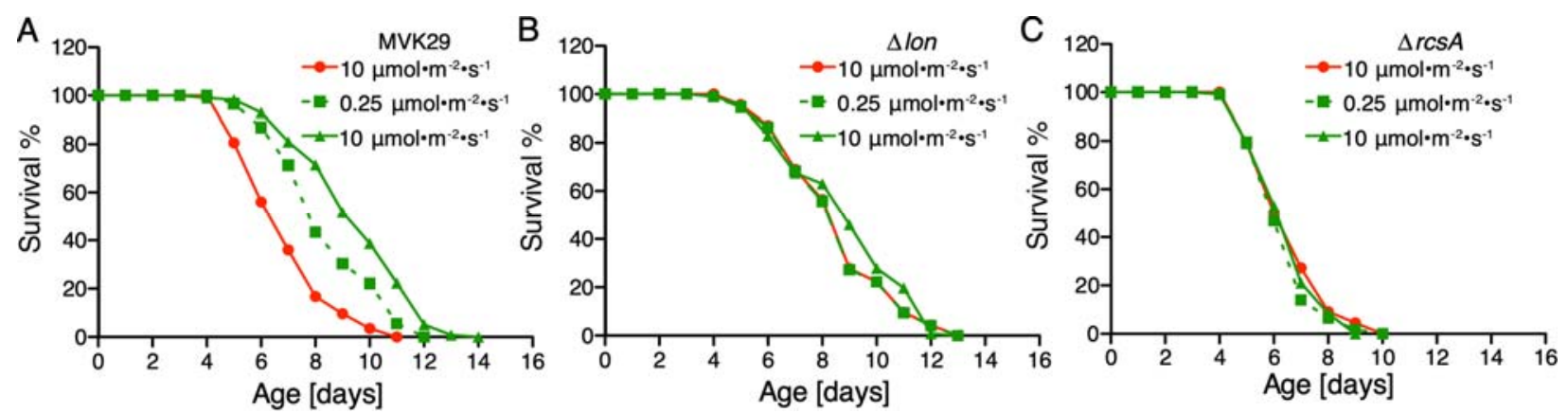

268 Figure 4. Optogenetically-regulated CA biosynthesis extends worm lifespan. (a) When

269 exposed to green light, worms grown on MVK29 live longer than those exposed to red light, and

270 the magnitude of lifespan extension is proportional to green light intensity $(p<0.0001$ green vs.

271 red, log-rank test). (b-c) The lifespans of worms grown on the $\Delta$ lon (b) or the $\Delta \operatorname{rcs} A$ (c) controls

272 are not affected by light condition $(p>0.1$ green vs. red, log-rank test). 


\section{Methods}

E. coli plasmids, strains, and media

Plasmids used in this study are described in Supplementary Table 1. Genbank accession

numbers are given in Supplementary Table 2. All plasmids constructed in this study were

279 assembled via Golden Gate cloning(Engler et al., 2009). Primers were ordered from IDT

280 (Coralville, IA). Assembled plasmids were transformed into E. coli NEB10ß (New England

281 Biolabs) for amplification and screening. All plasmid sequences were confirmed by Sanger

282 sequencing (Genewiz; S. Plainfield, NJ). To construct pLH401 and pLH405, pSR58.6(Schmidl

283 et al., 2014) was modified by inserting an mCherry expression cassette composed of a

284 constitutive promoter (J23114; http://parts.igem.org/Promoters/Catalog/Anderson), RBS

285 (BBa_B0034; http://parts.igem.org/Part:BBa_B0034), mCherry, and a synthetic transcriptional

286 terminator (L3S1P52 (Chen et al., 2013)). To construct pLH405, pLH401 was further modified

287 by exchanging the superfolder GFP gene ( $f f g f$ ) for $g f p m u t 3 *$. pMVK201.2 was built by 288 modifying pSR58.6 to control expression of $\operatorname{rcs} A$.

All E. coli strains are described in Supplementary Table 3. $\triangle$ rcsA (JW1935-1) was

290 obtained from the Coli Genetic Stock Center. $\Delta l o n$ (JW0429-1) was obtained from the Keio E.

291 coli knockout library(Baba et al., 2006), a gift from the Herman lab. All E. coli strains were

292 maintained in LB media supplemented with appropriate antibiotics (chloramphenicol $34 \mu \mathrm{g} / \mathrm{mL}$,

293 spectinomycin $100 \mu \mathrm{g} / \mathrm{mL}$, kanamycin $100 \mu \mathrm{g} / \mathrm{mL}$ ) in a shaking incubator at $37^{\circ} \mathrm{C}$ and $250 \mathrm{rpm}$

294 unless otherwise noted. 
C. elegans strains and media

All C. elegans strains (Supplementary Table 3) were provided by the Caenorhabditis

298 Genetics Center (University of Minnesota), which is funded by the NIH office of Research

299 Infrastructure Programs (P40 OD010440). Worms were grown at $20^{\circ} \mathrm{C}$ on $1.7 \%$ NGM-agar

300 plates in $60 \mathrm{~mm}$ Petri dishes inoculated with a lawn of E. coli (CGSC str. BW28357), as

301 described in the CGC WormBook (wormbook.org), unless otherwise specified. The common

302 strain E. coli OP50 was not used for worm feeding, as it produces CA during normal growth(Han

303 et al., 2017). M9 buffer for C. elegans (abbreviated M9Ce to distinguish from E. coli M9 media)

304 was composed of $3 \mathrm{~g} \mathrm{KH}_{2} \mathrm{PO}_{4}, 6 \mathrm{~g} \mathrm{Na}_{2} \mathrm{HPO}_{4}, 5 \mathrm{~g} \mathrm{NaCl}, 1 \mathrm{~mL} 1 \mathrm{M} \mathrm{MgSO}_{4}, \mathrm{H}_{2} \mathrm{O}$ to $1 \mathrm{~L}$, and

305 sterilized by autoclaving (wormbook.org).

307 Optogenetic control of CA production

$308 \quad 3 \mathrm{~mL}$ starter cultures of appropriate $E$. coli strains were inoculated from a $-80^{\circ} \mathrm{C}$ freezer

309 and grown $12 \mathrm{~h}$ at $37^{\circ} \mathrm{C}$. These starters were diluted to $\mathrm{OD}_{600}=1 \times 10^{-2}$ in $\mathrm{M} 9$ minimal media $(1 \mathrm{x}$

310 M9 salts, $0.4 \%$ w/v glucose, $0.2 \%$ w/v casamino acids, $\left.2 \mathrm{mM} \mathrm{MgSO}_{4}, 100 \mu \mathrm{M} \mathrm{CaCl}_{2}\right)$

311 supplemented with appropriate antibiotics. The M9/cell mixtures were then distributed into $3 \mathrm{~mL}$

312 aliquots in $15 \mathrm{~mL}$ clear polystyrene culture tubes and grown at $37^{\circ} \mathrm{C}$ in a shaking incubator at

$313250 \mathrm{rpm}$ while illuminated with the appropriate light wavelength and intensity, using the Light

314 Tube Array (LTA) device (Gerhardt et al., 2016). After 22 h, cultures were removed and iced to

315 halt growth and the $\mathrm{OD}_{600}$ was measured. Culture samples were collected for CA quantification. 


\section{CA quantification}

318 We adapted a previous CA quantification protocol(DISCHE, 1947; DISCHE and

319 SHETTLES, 1948) that takes advantage of the fact that it is the only exopolysaccharide

320 produced in our E. coli strains that incorporates fucose. In particular, we quantified the amount

321 of fucose in cell-derived exopolysaccharides (EPS), and used that value as a proxy CA levels.

322 First, EPS was liberated from cells by boiling $2 \mathrm{~mL}$ of culture for $15 \mathrm{~min}$. in a $15 \mathrm{~mL}$ conical

323 tube. The sample was then centrifuged in $1.5 \mathrm{~mL}$ Eppendorf tubes for $15 \mathrm{~min}$. at 21,000 x $\mathrm{g}$.

324 Then, $0.7 \mathrm{~mL}$ of supernatant was dialyzed against water for at least $12 \mathrm{~h}$ using Pur-A-Lyzer Midi

3253500 dialysis mini-tubes (Sigma-Aldrich, PURD35100-1KT) to remove monomeric fucose from

326 the sample.

Fucose monomers were then liberated from the EPS polymers by hydrolyzing $0.2 \mathrm{~mL}$ of

328 dialyzed media with $0.9 \mathrm{~mL}$ of $\mathrm{H}_{2} \mathrm{SO}_{4}$ solution (6:1 v/v acid:water). This mixture was boiled in a

$32915 \mathrm{~mL}$ conical for $20 \mathrm{~min}$ and then cooled to room temperature. The absorbance at $396 \mathrm{~nm}$ and

$330427 \mathrm{~nm}$ was measured. Next, $25 \mu \mathrm{L}$ of $1 \mathrm{M}$ L-cysteine $\mathrm{HCl}$ was added and mixed thoroughly by

331 pipetting. The absorbance at $396 \mathrm{~nm}$ and $427 \mathrm{~nm}$ was measured again. Simultaneously,

332 absorbance measurements of L-fucose standards pre- and post-L-cysteine addition were also

333 recorded. Absorbance change, given by $D$ in the formula below, were used to compare the L-

334 fucose standard samples to the dialyzed culture samples and estimate the L-fucose concentration

335 in the dialyzed product.

$$
D=\left(\left(A_{\text {post }}^{396}-A_{\text {pre }}^{396}\right)-\left(A_{\text {post }}^{427}-A_{\text {pre }}^{427}\right)\right)
$$


337 Preparation of NGM-agar plates for worm feeding

$338 \quad 3 \mathrm{~mL} \mathrm{E}$. coli starter cultures were inoculated from $-80^{\circ} \mathrm{C}$ freezer stocks and grown for 12

$339 \mathrm{~h}$ at $37^{\circ} \mathrm{C}$. These starters were then diluted to $\mathrm{OD}_{600}=1 \times 10^{-6}$ in $\mathrm{M} 9$ minimal media

340 supplemented with appropriate antibiotics. The M9/cell mixture was then distributed into $3 \mathrm{~mL}$

341 aliquots in $15 \mathrm{~mL}$ clear polystyrene culture tubes and grown at $37^{\circ} \mathrm{C}$ in a shaking incubator at

$342250 \mathrm{rpm}$ while illuminated with the appropriate light in the LTA. Once cultures reached $\mathrm{OD}_{600}=$

$3430.1-0.4$, tubes were iced for $10 \mathrm{~min}$ and subsequently concentrated to $\mathrm{OD}_{600} \sim 20$ by

344 centrifugation $\left(4^{\circ} \mathrm{C}, 4000 \mathrm{rpm}, 10 \mathrm{~min}\right)$ and resuspension in fresh M9 media. $400-600 \mu \mathrm{L}$ of

345 dense bacterial culture was then applied to sterile NGM-agar plates and allowed to dry in a dark

346 room, or a room with green overhead safety lights if cultures were preconditioned in green light.

347 Plates were wrapped in foil and refrigerated at $4{ }^{\circ} \mathrm{C}$ for no more than 5 days until needed.

349 Time-lapse microscopy

350 To obtain age-synchronized worm cultures, axenized C. elegans (strain glo-1) eggs were

351 isolated and allowed to arrest in L1 by starvation in M9 buffer (distinct from M9 media: $3 \mathrm{~g}$

$352 \mathrm{KH}_{2} \mathrm{PO}_{4}, 6 \mathrm{~g} \mathrm{Na}_{2} \mathrm{HPO}_{4}, 5 \mathrm{~g} \mathrm{NaCl}, 1 \mathrm{~mL} 1 \mathrm{M} \mathrm{MgSO}_{4}$, and water to $1 \mathrm{~L}$, sterilized by autoclaving

353 at $121^{\circ} \mathrm{C}$ for $20 \mathrm{~min}$ ) at room temperature for $12-18 \mathrm{~h} \cdot 10-100$ larvae were transferred to a

354 previously prepared NGM-agar plate containing a lawn of the appropriate bacterial strain. The

355 plate was then placed in a $20^{\circ} \mathrm{C}$ incubator and illuminated with appropriate optogenetic light

356 provided by a single LED positioned $1 \mathrm{~cm}$ above the Petri dish. Adult worms were transferred to

357 a fresh plate as necessary to maintain only a single generation. 
Individual worms aged 1-3 days were removed from the dish and prepared for time-lapse

359 epifluorescence imaging. A $1.5 \%$ agar pad was prepared using M9 buffer as previously

360 described $^{45}$, and punched into 1/2" circles with a hollow punch. A $4 \mu \mathrm{L}$ droplet of $2 \mathrm{mM}$

361 levamisole was deposited on a pad and 5 adult worms were transferred from the NGM plate to

362 the droplet. An additional $4 \mu \mathrm{L}$ of levamisole solution was added and the worms were gently

363 washed to remove external bacteria. Worms were then transferred to a fresh pad with a $4 \mu \mathrm{L}$

364 droplet of levamisole solution, which was allowed to dry, thereby co-localizing and aligning the

365 worms on the pad. The pad was then inverted and placed into a $13 \mathrm{~mm}$ disposable microscopy

366 petri dish with a \#1.5 coverslip on the bottom (Cell E\&G; Houston, TX). Another coverslip was

367 placed on the top of the pad in the dish to curtail evaporation.

The dish was then placed on the stage of a Nikon Eclipse Ti-E inverted epifluorescence

microscope (Nikon Instruments, Inc; Melville, NY). Complete paralysis was induced by

370 incubating the dish at room temperature $\left(\sim 23^{\circ} \mathrm{C}\right)$ for $30 \mathrm{~min}$. Meanwhile, worms were exposed

371 to appropriate preconditioning light supplied by a circular array of 8 LEDs $(4 \times 660 \mathrm{~nm}, 4 \times 525$

$372 \mathrm{~nm}$ ) mounted to the microscope condenser ring, about $2 \mathrm{~cm}$ above the Petri dish. Light was then

373 switched from the preconditioning to the experimental wavelength, and worms were imaged

374 periodically using 10x, 40x, and 60x objectives. For each time point, the LEDs were turned off

375 and images acquired in the brightfield (DIC) and fluorescent channels. Afterwards, the LEDs

376 were turned on again to maintain optogenetic control.

$378 \quad$ Epifluorescence image analysis

379 All epifluorescence images were analyzed using the Nikon Elements software package

380 (Nikon Instruments, Inc; Melville, NY). The mCherry signal was used as a marker for the gut 
381 lumen, and only cells in this region were included in the analysis. Image ROIs were created by

382 thresholding the sfGFP signal to identify the boundaries of cell populations. Out of focus regions

383 were eliminated from analysis. The average sfGFP pixel intensity inside the ROIs was calculated

384 and recorded for each time point.

Flow cytometry

1-3 day old glo-1 worms were prepared for flow cytometry of the microbiome constituents by washing, using a protocol adapted from previous work(Portal-Celhay et al.,

390 buffer containing $2 \mathrm{mM}$ levamisole, $1 \%$ Triton $\mathrm{X}-100$, and $100 \mathrm{mg} / \mathrm{mL}$ ampicillin. The worms

391 were then washed $2 x$ in $5 \mu \mathrm{L}$ droplets of M9 buffer containing 2 mM levamisole only. Finally,

392 the worms were transferred to clear $0.5 \mathrm{~mL}$ Eppendorf tubes containing $50 \mu \mathrm{L}$ of M9 buffer +2

$393 \mathrm{mM}$ levamisole, ensuring that 5 worms were deposited in the liquid contained in each tube. Each

394 tube was then exposed to light by placing it within one well of a 24-well plate (AWLS-303008,

395 ArcticWhite LLC) atop a Light Plate Apparatus (LPA) containing green and red LEDs ${ }^{47}$ for $8 \mathrm{~h}$

396 at room temperature. In separate control experiments, we demonstrated that any stray bacteria

397 that may escape the worms over this period, or which were inadvertently added to the $50 \mu \mathrm{L}$ of

398 M9 buffer, are incapable of responding to optogenetic light (Supplementary Fig. 4).

At the conclusion of the experiment, tubes were removed from the plate and immediately

400 chilled in an ice slurry for $10 \mathrm{~min}$ in the dark. Worms were homogenized using an anodized steel

401 probe sterilized between samples via $70 \%$ ethanol treatment and flame before being cooled. 
Next, we used our previous antibiotic-based fluorescent protein maturation

403 protocol(Olson et al., 2014) to allow unfolded proteins to mature while preventing the production

404 of new protein. In particular, $250 \mu \mathrm{L}$ PBS containing $500 \mathrm{mg} / \mathrm{mL}$ Rifampicin was added to the

$40550 \mu \mathrm{L}$ homogenized worm samples and transferred to cytometry tubes. These tubes were

406 incubated in a $37^{\circ} \mathrm{C}$ water bath for precisely $1 \mathrm{~h}$, then transferred back to an ice slurry.

These samples were measured on a BD FACScan flow cytometer. For gating, an

408 FSC/SSC polygon gate was first created using non-fluorescent bacteria grown in vitro at $37^{\circ} \mathrm{C}$

409 (Supplementary Fig. 2). Events outside this region were excluded as non-bacterial material. To

410 isolate the engineered gut bacteria, only events with a high mCherry signal (FL3 > 1200 a.u.,

411 FL3 gain: 999) were included (Supplementary Fig. 2). Samples were measured until 20,000

412 events were recorded or the sample was exhausted.

$414 \quad$ Flow cytometry data analysis

415 All flow cytometry data (FCS format) were analyzed using FlowCal(Castillo-Hair et al.,

416 2016) and Python 2.7. We wrote a standard cytometry analysis workflow that truncated the

417 initial and final 10 events to prevent cross-sample contamination, removed events from saturated

418 detector bins at the ends of the detection range, and added 2D density gate on SSC/FSC retaining

419 the densest $75 \%$ of events (Supplementary Fig. 2a). GFPmut3* fluorescence units were

420 converted into standardized units of molecules of equivalent fluorescein (MEFL) using a

421 fluorescent bead standard (Rainbow calibration standard; cat. no. RCP-30-20A, Spherotech, Inc.)

422 as described previously(Castillo-Hair et al., 2016). Finally, to eliminate events associated with $C$.

423 elegans autofluorescence (Supplementary Fig. 2b), any events in the region FL1 $\leq 1200$ MEFL

424 were discarded. 
Synchronized L1 worms (strain ges-1) were applied to NGM agar plates containing bacterial strain BW25113 and allowed to develop until adulthood. This parental bacterial strain is

429 used to allow all worms to develop at the same rate, avoiding any developmental/growth effects

430 the experimental strains may exert on the worms. All experimental bacterial strains were

431 preconditioned in red light with optogenetic light provided by a single LED positioned $1 \mathrm{~cm}$ 432 above the Petri dish.

After 3-5 days (between days 1-3 of adulthood), worms allocated for the experiment were

434 transferred to experimental strains for approximately 60-90 minutes to thoroughly inoculate the

435 GI tract. In the case of the unparalyzed worms, red or green light was then applied for an

436 additional 6 hours. For the paralyzed worm experiments, 1.5\% low-melt agar pads were prepared

437 as described above and placed on individual slides. About 15 adult worms were transferred from

438 the experimental strain Petri dish to an agar pad containing $10 \mu \mathrm{L}$ of C. elegans M9 buffer +2

$439 \mathrm{mM}$ levamisole (M9Ce+Lev), where worms were gently washed before being transferred to a

440 fresh pad also containing $10 \mu \mathrm{L}$ of M9Ce+Lev. The majority of M9Ce+Lev on the pad was

441 allowed to evaporate, which causes the worms to align longitudinally before a cover slip was

442 applied. Slides were then exposed to either red or green light by placing them under a single

443 LED positioned $1 \mathrm{~cm}$ above the Petri dish for $6 \mathrm{~h}$. Afterward, the anterior intestinal cells were

444 imaged using confocal microscopy (Olympus Fluoview 3000) in the brightfield and GFP 445 channels. 


\section{Confocal Microscopy Image Analysis}

448 All confocal images for an experiment were manually cropped to display only the 449 anterior intestinal cells of a single worm (in the GFP channel). These cropped images were then

450 anonymized, randomized and the mitochondrial networks of each were blindly classified by two

451 researchers independently as either tubular, fragmented, or intermediate. Tubular samples were

452 marked by a high degree of network connectivity throughout. Fragmented samples were 453 composed almost exclusively of isolated clusters of fluorescence with high circularity.

454 Intermediate samples contained regions of both types. Scores were then de-randomized and 455 aggregated. For each experimental strain, the red and green light conditions were compared for 456 statistical significance using the chi-squared test of homogeneity.

\section{$458 \quad$ Lifespan experiments}

$4593 \mathrm{~mL}$ starter cultures of $\triangle l o n, \Delta r c s A$ or MVK29 were inoculated from $-80^{\circ} \mathrm{C}$ freezer 460 stocks into LB supplemented with appropriate antibiotics and grown shaking for $12 \mathrm{~h}$ at $37^{\circ} \mathrm{C}$ at $461250 \mathrm{rpm}$. These cultures were diluted to $\mathrm{OD}_{600}=1 \times 10^{-6}$ in $27 \mathrm{~mL}$ M9 media supplemented with 462 appropriate antibiotics. $1.5 \mathrm{~mL}$ of each M9/cell mixture was added to each of 18 wells on three 463 24-well plates and grown in 3 LPA devices under the appropriate light conditions at $37{ }^{\circ} \mathrm{C}$ and $464250 \mathrm{rpm}$. Once cultures reached $\mathrm{OD}_{600}=0.1-0.4$, all tubes were iced for $10 \mathrm{~min}$ and subsequently 465 concentrated 10 times by centrifugation $\left(4^{\circ} \mathrm{C}, 4000 \mathrm{rpm}, 10 \mathrm{~min}\right)$. Approximately $50 \mu \mathrm{L}$ of this 466 dense bacterial culture was then applied to sterile NGM-agar plates with no antibiotics and 467 allowed to dry in a dark room. The plates were then illuminated with the appropriate light 468 wavelength and intensity for $16 \mathrm{~h}$ at room temperature, and immediately used for lifespan assays. 
470 level. To reach this goal, the sqt-3(e2117) temperature sensitive mutant (Supplementary Table

471 3) was used to perform longitudinal analyses at $25^{\circ} \mathrm{C}$, which avoids time-consuming animal

472 transfer without interrupting normal reproduction. sqt-3(e2117) is a collagen mutant of $C$.

473 elegans that reproduces normally but is embryonically lethal at $26{ }^{\circ} \mathrm{C}$, and has been used

474 previously in longitudinal studies(Han et al., 2017; Wang et al., 2014). Worms were age-

475 synchronized by bleach-based egg isolation followed by starvation in M9 buffer at the L1 stage

476 for 36 hours. Synchronized L1 worms were grown on BW25113 E. coli at $15^{\circ} \mathrm{C}$ until the L4

477 stage, when worms were transferred to 24 -well plates ( 15 worms/well) with $\Delta l o n, \Delta r c s A$ or

478 MVK29 (Supplementary Table 3). The plates were placed in LPA. The LPA LEDs were

479 programmed to illuminate wells with constant red $\left(10 \mu \mathrm{mol} / \mathrm{m}^{2} / \mathrm{s}\right)$, low-intensity green $(0.25$

$\left.480 \mu \mathrm{mol} / \mathrm{m}^{2} / \mathrm{s}\right)$, or high-intensity green light $\left(10 \mu \mathrm{mol} / \mathrm{m}^{2} / \mathrm{s}\right)$. The apparatus was then transferred to a

$48126{ }^{\circ} \mathrm{C}$ incubator. The number of living worms remaining in each well was counted every day.

482 Death was indicated by total cessation of movement in response to gentle mechanical

483 stimulation. Statistical analyses were performed with SPSS (IBM Software) using Kaplan-Meier

484 survival analysis and the log-rank test (Supplementary Table 4).

486 Supplemental Information Titles and Legends

487 Supplementary Figure 1. In vitro characterization of GFP reporter strains used in this

488 study. All optogenetic strains carry our previously published CcaSR v2.0 system, which is 489 encoded on plasmids (a) pSR43.6 and (b) pSR58.6. (c) pLH401 (used for microscopy) and (d)

490 pLH405 (used for cytometry) genetic device schematics. We replaced sfgfp with $g f p m u t 3 *$ in 491 pLH405 as we hypothesized that the latter may be less stable and thus result in faster response 
492 dynamics. However, we observed no difference in dynamics. (e) Batch culture light responses of

493 CcaSR v2.0 and all GFP reporter strains used in this study. GFP fluorescence was measured by

494 flow cytometry. The dynamic range (ratio of GFP fluorescence in green versus red light) is

495 shown above each data set. We note that CcaSR v2.0 exhibits 77-fold dynamic range in the

496 reference strain BW29655 ( $\triangle e n v Z, \Delta o m p R)$, which is similar to our previous measurement of

497 this strain at 120-fold (Schmidl et al., 2014). The calculated fold-change is sensitive to

498 fluctuations in the measured E. coli autofluorescence and red-light expression level, which likely

499 explains the slight discrepancy. CcaSR v2.0 dynamic range increases slightly to 84.2-fold in

$500 \Delta r c s A$ (JW1935-1), which is used throughout this work, due to higher sfGFP expression in green

501 light. The mCherry cassette in pLH401 decreases dynamic range to 38.3-fold due to higher leaky

502 sfGFP expression in red light. In worms, the fold-change in response to green light decreases

503 further to $5.52 \pm 2.4$-fold (Fig. 1d). The use of gfpmut $3 *$ in pLH405 further decreases dynamic

504 range to $13.5 \pm 0.0$-fold for reasons that are not clear, while in worms the fold-change is $8.63 \pm$

505 3.6-fold (Fig. 1e). Data represent the mean of three independent, autofluorescence-subtracted,

506 biological replicates acquired on 3 separate days. Error bars: standard deviation.

508 Supplementary Figure 2. Flow cytometry gating. (a) Strain LH05 (Supplementary Table 3),

509 which expresses only mCherry, was fed to worms, isolated, and analyzed by flow cytometry to

510 quantify E. coli autofluorescence through the FL1 (GFP) channel. To eliminate events

511 corresponding to cytometer noise and autofluorescence of homogenized worm samples, three

512 gates were then applied: (1) a density gate for the most homogeneous $75 \%$ of samples in forward

513 scatter (FSC) vs side-scatter (SSC) (the area within the bold black line in the plots in Column 1),

514 (2) a threshold gate on the FL1 (GFP) channel (marked by a red dashed line; Methods), and (3) a 
515 threshold gate for events exhibiting high FL3 (mCherry, red dashed line; Methods). (b)

516 Applying these gates to samples from the step ON experiment (Fig. 1e) reveals robust isolation

517 of bacteria with a FSC/SSC profile consistent with our previous in vitro experiments ${ }^{24,47}$ and an

518 increase in expression of GFPmut $3 *$ in response to green light. Ungated data are shown for 519 comparison to gated data in all plots.

521 Supplementary Figure 3. Gene expression dynamics from flow cytometry experiments. (a)

522 LH03 and (b) LH04 ( $\triangle \mathrm{PCB})$ step ON results. (c) LH03 and (d) LH04 step OFF results. Each

523 individual trajectory (faint lines) corresponds to a single biological replicate. A biological

524 replicate comprises the homogenized contents of five worms collected on a single day. Each

525 biological replicate on a given plot was run on a different day. Population medians taken across

526 all trajectories are shown in bold (Methods). Data are composed of six and eight trajectories in

527 the step ON and step OFF experiments, respectively. Error bars: SEM.

529 Supplementary Figure 4. Bacteria on the exterior of worms do not contribute to the

530 measured light response in flow cytometry experiments. Worms were suspended in clear

531 tubes containing M9Ce+Lev media and levamisole in the flow cytometry experiments. To

532 demonstrate that bacterial cells outside the worm gut (e.g. on the exterior of the worm) do not

533 respond to light in these conditions, samples of pre-conditioned bacteria from the NGM plates

534 were suspended in the M9Ce+Lev or E. coli M9 media supplemented with casamino acids and

535 glucose (M9Ec). These samples were then exposed to either green or red light for $8 \mathrm{~h}$, then

536 measured via flow cytometry after the fluorophore maturation protocol (Methods). As expected, 
537 the bacterial populations are only responsive to light when grown in M9Ec and when the PCB

538 operon is intact (strain JW1935-1/pLH405/pSR43.6, aka LH03). We conclude that the responses

539 shown in data in Fig. 1e-f and Supplementary Fig. 3 are not due to bacteria outside the worm,

540 nor to bacteria that escape the worm over the course of the experiment, as such cells do not

541 respond to light in the experimental buffer (M9Ce). Data represent 7, 6, 3, and 5 replicates (left);

542 and 8, 3, 8, and 3 replicates (right) over 8 and 10 days, respectively; error bars: SEM. Individual

543 data points for each condition are overlaid as white markers.

\section{Supplementary Table 1. Plasmids used in this study}

545 Supplementary Table 2. Genbank Accession Numbers

546 Supplementary Table 3. Bacterial and worm strains used in this study

547 Supplementary Table 4. Statistical analysis of worm lifespan experiments

549 References

551 Baba T, Ara T, Hasegawa M, Takai Y, Okumura Y, Baba M, Datsenko KA, Tomita M, Wanner

552 BL, Mori H. 2006. Construction of Escherichia coli K $\square 12$ in $\square$ frame, single $\square$ gene knockout 553 mutants: the Keio collection. Mol Syst Biol 2:2006.0008. doi:10.1038/msb4100050

554 Biagi E, Franceschi C, Rampelli S, Severgnini M, Ostan R, Turroni S, Consolandi C, Quercia S, 555 Scurti M, Monti D, Capri M, Brigidi P, Candela M. 2016. Gut Microbiota and Extreme 556 Longevity. Curr Biol 26:1480-1485. doi:10.1016/j.cub.2016.04.016 
557 Cabreiro F, Au C, Leung K-Y, Vergara-Irigaray N, Cochemé HM, Noori T, Weinkove D, 558 Schuster E, Greene NDE, Gems D. 2013. Metformin retards aging in C. elegans by altering 559 microbial folate and methionine metabolism. Cell 153:228-39. doi:10.1016/j.cell.2013.02.035

560 Castillo-Hair SM, Baerman EA, Fujita M, Igoshin OA, Tabor JJ. 2019. Optogenetic control of 561 Bacillus subtilis gene expression. Nat Commun 10:3099. doi:10.1038/s41467-019-10906-6

562 Castillo-Hair SM, Sexton JT, Landry BP, Olson EJ, Igoshin OA, Tabor JJ. 2016. FlowCal: A 563 User-Friendly, Open Source Software Tool for Automatically Converting Flow Cytometry 564 Data from Arbitrary to Calibrated Units. Acs Synth Biol 5:774-80. 565 doi:10.1021/acssynbio.5b00284

566 Chait R, Ruess J, Bergmiller T, Tkačik G, Guet CC. 2017. Shaping bacterial population behavior 567 through computer-interfaced control of individual cells. Nat Commun 8:1535. doi:10.1038/s41467-017-01683-1

569 Chen Y-J, Liu P, Nielsen AAK, Brophy JAN, Clancy K, Peterson T, Voigt CA. 2013. 570 Characterization of 582 natural and synthetic terminators and quantification of their design $571 \quad$ constraints. Nat Methods 10:659-664. doi:10.1038/nmeth.2515

572 Cho D-H, Nakamura T, Fang J, Cieplak P, Godzik A, Gu Z, Lipton SA. 2009. S-nitrosylation of 573 Drp1 mediates beta-amyloid-related mitochondrial fission and neuronal injury. Sci New York N Y 324:102-5. doi:10.1126/science.1171091

575 Claesson MJ, Cusack S, O’Sullivan O, Greene-Diniz R, Weerd H de, Flannery E, Marchesi JR, 576 Falush D, Dinan T, Fitzgerald G, Stanton C, Sinderen D van, O'Connor M, Harnedy N, 577 O’Connor K, Henry C, O’Mahony D, Fitzgerald AP, Shanahan F, Twomey C, Hill C, Ross 578 RP, O'Toole PW. 2010. Composition, variability, and temporal stability of the intestinal 
microbiota of the elderly. P Natl Acad Sci Usa 108 Suppl 1:4586-91. doi:10.1073/pnas.1000097107 M, Lakshminarayanan B, O’Sullivan O, Fitzgerald GF, Deane J, O’Connor M, Harnedy N, O’Connor K, O’Mahony D, Sinderen D van, Wallace M, Brennan L, Stanton C, Marchesi JR, Fitzgerald AP, Shanahan F, Hill C, Ross RP, O’Toole PW. 2012. Gut microbiota composition correlates with diet and health in the elderly. Nature 488:178-84. doi:10.1038/nature11319 Immun 70:4705-4707. doi:10.1128/iai.70.8.4705-4707.2002 18:1213-25. doi:10.1038/nn.4091 98.

592 DISCHE Z, SHETTLES LB. 1948. A specific color reaction of methylpentoses and a 593 spectrophotometric micromethod for their determination. J Biological Chem 175:595-603.

594 Donato V, Ayala FR, Cogliati S, Bauman C, Costa JG, Leñini C, Grau R. 2017. Bacillus subtilis 595 biofilm extends Caenorhabditis elegans longevity through downregulation of the insulin-like 596 signalling pathway. Nat Commun 8:14332. doi:10.1038/ncomms14332

597 Engler C, Gruetzner R, Kandzia R, Marillonnet S. 2009. Golden gate shuffling: a one-pot DNA 598 shuffling method based on type IIs restriction enzymes. Plos One 4:e5553. 599 doi:10.1371/journal.pone.0005553 
600 Exner N, Treske B, Paquet D, Holmstrom K, Schiesling C, Gispert S, Carballo-Carbajal I, Berg 601 D, Hoepken H-H, Gasser T, Kruger R, Winklhofer KF, Vogel F, Reichert AS, Auburger G, 602 Kahle PJ, Schmid B, Haass C. 2007. Loss-of-Function of Human PINK1 Results in 603 Mitochondrial Pathology and Can Be Rescued by Parkin. J Neurosci 27:12413-12418. doi:10.1523/jneurosci.0719-07.2007

605 Fernandez-Rodriguez J, Moser F, Song M, Voigt CA. 2017. Engineering RGB color vision into Escherichia coli. Nat Chem Biol 13:706-708. doi:10.1038/nchembio.2390

607 Gautier A, Gauron C, Volovitch M, Bensimon D, Jullien L, Vriz S. 2014. How to control 608 proteins with light in living systems. Nat Chem Biol 10:533-541. doi:10.1038/nchembio.1534

609 Gerhardt KP, Olson EJ, Castillo-Hair SM, Hartsough LA, Landry BP, Ekness F, Yokoo R, 610 Gomez EJ, Ramakrishnan P, Suh J, Savage DF, Tabor JJ. 2016. An open-hardware platform 611 for optogenetics and photobiology. Sci Rep-uk 6:35363. doi:10.1038/srep35363

612 Goglia AG, Toettcher JE. 2018. A bright future: optogenetics to dissect the spatiotemporal 613 control of cell behavior. Curr Opin Chem Biol 48:106-113. doi:10.1016/j.cbpa.2018.11.010

614 Gusarov I, Gautier L, Smolentseva O, Shamovsky I, Eremina S, Mironov A, Nudler E. 2013. 615 Bacterial Nitric Oxide Extends the Lifespan of C. elegans. Cell 152:818-830. 616 doi:10.1016/j.cell.2012.12.043

617 Han B, Sivaramakrishnan P, Lin C-CJ, Neve IAA, He J, Tay LWR, Sowa JN, Sizovs A, Du G, 618 Wang J, Herman C, Wang MC. 2017. Microbial Genetic Composition Tunes Host Longevity. 619 Cell 169:1249-1262.e13. doi:10.1016/j.cell.2017.05.036

620 Kenyon CJ. 2010. The genetics of ageing. Nature 464:504-512. doi:10.1038/nature08980 
621 Kim DH. 2013. Bacteria and the aging and longevity of Caenorhabditis elegans. Annu Rev Genet 47:233-46. doi:10.1146/annurev-genet-111212-133352

623 Köhler P, Bachmann R. 1978. The effects of the antiparasitic drugs levamisole, thiabendazole, 624 praziquantel, and chloroquine on mitochondrial electron transport in muscle tissue from 625 Ascaris suum. Mol Pharmacol 14:155-63.

626 Kotula JW, Kerns SJ, Shaket LA, Siraj L, Collins JJ, Way JC, Silver PA. 2014. Programmable 627 bacteria detect and record an environmental signal in the mammalian gut. P Natl Acad Sci Usa 111:4838-43. doi:10.1073/pnas.1321321111

629 Kundu P, Blacher E, Elinav E, Pettersson S. 2017. Our Gut Microbiome: The Evolving Inner Self. Cell 171:1481-1493. doi:10.1016/j.cell.2017.11.024

631 Leopold AV, Chernov KG, Verkhusha VV. 2018. Optogenetically controlled protein kinases for 632 regulation of cellular signaling. Chem Soc Rev 47:2454-2484. doi:10.1039/c7cs00404d

633 Levskaya A, Chevalier AA, Tabor JJ, Simpson ZB, Lavery LA, Levy M, Davidson EA, Scouras 634 A, Ellington AD, Marcotte EM, Voigt CA. 2005. Engineering Escherichia coli to see light. $635 \quad$ Nature 438:441-442. doi:10.1038/nature04405

636 Li X, Zhang C, Xu X, Miao J, Yao J, Liu R, Zhao Y, Chen X, Yang Y. 2020. A single637 component light sensor system allows highly tunable and direct activation of gene expression 638 in bacterial cells. Nucleic Acids Res. doi:10.1093/nar/gkaa044

639 Lim B, Zimmermann M, Barry NA, Goodman AL. 2017. Engineered Regulatory Systems 640 Modulate Gene Expression of Human Commensals in the Gut. Cell 169:547-558.e15. $641 \quad$ doi:10.1016/j.cell.2017.03.045 
642 Milias-Argeitis A, Rullan M, Aoki SK, Buchmann P, Khammash M. 2016. Automated 643 optogenetic feedback control for precise and robust regulation of gene expression and cell 644 growth. Nat Commun 7:12546. doi:10.1038/ncomms 12546

645 Mimee M, Tucker AC, Voigt CA, Lu TK. 2015. Programming a Human Commensal Bacterium, 646 Bacteroides thetaiotaomicron, to Sense and Respond to Stimuli in the Murine Gut Microbiota. $647 \quad$ Cell Syst 1:62-71. doi:10.1016/j.cels.2015.06.001

648 Ohlendorf R, Vidavski RR, Eldar A, Moffat K, Möglich A. 2012. From Dusk till Dawn: One649 Plasmid Systems for Light-Regulated Gene Expression. J Mol Biol 416:534-542. $650 \quad$ doi:10.1016/j.jmb.2012.01.001

651 Olson EJ, Hartsough LA, Landry BP, Shroff R, Tabor JJ. 2014. Characterizing bacterial gene 652 circuit dynamics with optically programmed gene expression signals. Nat Methods 11:449$653 \quad 55$. doi:10.1038/nmeth.2884

654 Olson EJ, Tabor JJ. 2014. Optogenetic characterization methods overcome key challenges in 655 synthetic and systems biology. Nat Chem Biol 10:502-11. doi:10.1038/nchembio.1559

656 Olson EJ, Tzouanas CN, Tabor JJ. 2017. A photoconversion model for full spectral 657 programming and multiplexing of optogenetic systems. Mol Syst Biol 13:926. 658 doi:10.15252/msb.20167456

659 Ong NT, Tabor JJ. 2018. A Miniaturized Escherichia coli Green Light Sensor with High 660 Dynamic Range. Chembiochem 19:1255-1258. doi:10.1002/cbic.201800007

661 Ong NTX, Olson EJ, Tabor JJ. 2017. Engineering an E. coli near-infrared light sensor. Acs Synth 662 Biol 7:240-248. doi:10.1021/acssynbio.7b00289 
663 O'Toole PW, Jeffery IB. 2015. Gut microbiota and aging. Sci New York N Y 350:1214-5.

664 doi:10.1126/science.aac8469

665 Portal-Celhay C, Bradley ER, Blaser MJ. 2012. Control of intestinal bacterial proliferation in 666 regulation of lifespan in Caenorhabditis elegans. Bmc Microbiol 12:49. doi:10.1186/1471$667 \quad 2180-12-49$

668 Pryor R, Norvaisas P, Marinos G, Best L, Thingholm LB, Quintaneiro LM, Haes WD, Esser D, 669 Waschina S, Lujan C, Smith RL, Scott TA, Martinez-Martinez D, Woodward O, Bryson K, 670 Laudes M, Lieb W, Houtkooper RH, Franke A, Temmerman L, Bjedov I, Cochemé HM, 671 Kaleta C, Cabreiro F. 2019. Host-Microbe-Drug-Nutrient Screen Identifies Bacterial 672 Effectors of Metformin Therapy. Cell 178:1299-1312.e29. doi:10.1016/j.cell.2019.08.003

673 Ramakrishnan P, Tabor JJ. 2016. Repurposing Synechocystis PCC6803 UirS-UirR as a UV-

674 Violet/Green Photoreversible Transcriptional Regulatory Tool in E. coli. Acs Synth Biol $675 \quad$ 5:733-40. doi:10.1021/acssynbio.6b00068

676 Ryu M-H, Gomelsky M. 2014. Near-infrared light responsive synthetic c-di-GMP module for 677 optogenetic applications. Acs Synth Biol 3:802-10. doi:10.1021/sb400182x

678 Schmidl SR, Sheth RU, Wu A, Tabor JJ. 2014. Refactoring and Optimization of Light679 SwitchableEscherichia coliTwo-Component Systems. Acs Synth Biol 3:820-831. 680 doi:10.1021/sb500273n

681 Sebastián D, Palacín M, Zorzano A. 2017. Mitochondrial Dynamics: Coupling Mitochondrial 682 Fitness with Healthy Aging. Trends Mol Med 23:201-215. 683 doi:10.1016/j.molmed.2017.01.003 
684 Tabor JJ, Levskaya A, Voigt CA. 2010. Multichromatic control of gene expression in 685 Escherichia coli. J Mol Biol 405:315-24. doi:10.1016/j.jmb.2010.10.038

686 Tabor JJ, Salis HM, Simpson ZB, Chevalier AA, Levskaya A, Marcotte EM, Voigt CA,

687 Ellington AD. 2009. A synthetic genetic edge detection program. Cell 137:1272-81. 688 doi:10.1016/j.cell.2009.04.048

689 Tandar ST, Senoo S, Toya Y, Shimizu H. 2019. Optogenetic switch for controlling the central 690 metabolic flux of Escherichia coli. Metab Eng 55:68-75. doi:10.1016/j.ymben.2019.06.002

691 Virk B, Jia J, Maynard CA, Raimundo A, Lefebvre J, Richards SA, Chetina N, Liang Y, 692 Helliwell N, Cipinska M, Weinkove D. 2016. Folate Acts in E. coli to Accelerate C. elegans 693 Aging Independently of Bacterial Biosynthesis. Cell Reports 14:1611-20. 694 doi:10.1016/j.celrep.2016.01.051

695 Wang MC, Oakley HD, Carr CE, Sowa JN, Ruvkun G. 2014. Gene pathways that delay 696 Caenorhabditis elegans reproductive senescence. Plos Genet 10:e1004752. 697 doi:10.1371/journal.pgen.1004752

698 Zhang F, Berg M, Dierking K, Félix M-A, Shapira M, Samuel BS, Schulenburg H. 2017. 699 Caenorhabditis elegans as a Model for Microbiome Research. Front Microbiol 8:485. 700 doi:10.3389/fmicb.2017.00485 